\title{
Spin Torque in Double Planar Tunnel Junctions
}

\author{
M. WilCZYŃSKI*, R. ŚWIRKOWICZ \\ Faculty of Physics, Warsaw University of Technology \\ Koszykowa 75, 00-662 Warsaw, Poland \\ AND J. BARNAŚ \\ Department of Physics, Adam Mickiewicz University \\ Umultowska 85, 61-614 Poznań, Poland \\ and Institute of Molecular Physics, Polish Academy of Sciences \\ M. Smoluchowskiego 17, 60-179 Poznań, Poland
}

\begin{abstract}
Transport in a double planar tunnel junction with ferromagnetic electrodes is analyzed theoretically in the zero-temperature limit. The in-plane and out-of-plane components of the spin torque exerted on magnetic moment of the central layer are determined as a function of the angle $\theta$ between magnetic moments of one of the external electrodes and of the central layer. Two configurations of the magnetic moments of external electrodes are considered, i.e. parallel and antiparallel ones. It is found that both torque components depend strongly on the thickness of the central layer, especially in junctions with a relatively thin central layer, where enhancement of the torque or a change of its sign can be observed for specific layer thicknesses. In junctions with thick central layer and in the limit of small bias voltage applied to the junction, the in-plane torque is generally smaller in the parallel configuration than in the antiparallel one. The opposite relation is observed for the normal torque.
\end{abstract}

PACS numbers: 73.40.Gk, 72.25.-b

\section{Introduction}

Electrons tunneling through a barrier between two ferromagnetic electrodes with non-collinear magnetic moments can exert a torque on the magnetic moments of both electrodes. This torque (called spin torque) is a consequence of the spin transfer from conduction electrons to localized magnetic moments [1]. It has been found experimentally that a sufficiently large spin torque can lead to switching of the magnetic moments $[2,3]$, which is promising for various applications, e.g. in magnetic memory elements. However, to obtain the torque that is sufficient to rotate magnetic moment, the current density of an order $10^{5}-10^{7} \mathrm{~A} / \mathrm{cm}^{2}$ is required. The high bias voltage should be then applied to the junction, which however is not good from the application point of view due to possible electrical breakdown of the tunnel barrier. The enhancement of the tunneling current and consequently of the spin torque in a region of low bias voltage can be obtained in the double junction due to the formation of resonant states in the central layer [4-6].

\section{Model}

In this paper we consider a double planar tunnel junction with three ferromagnetic layers and two nonmagnetic barriers (Fig. 1). External magnetic layers are

\footnotetext{
* corresponding author; e-mail: wilczyns@if.pw.edu.pl
}

assumed to be thick enough to be considered as semi-infinite electrodes. It is also assumed that magnetic moments of all three layers are in the junction plane. We consider two collinear configurations of magnetic moments of the external electrodes. In the parallel configuration magnetic moments of the left and right electrodes, $S^{\mathrm{l}}$ and $S^{\mathrm{r}}$, point in the same direction, whereas in the antiparallel configuration they are opposite.

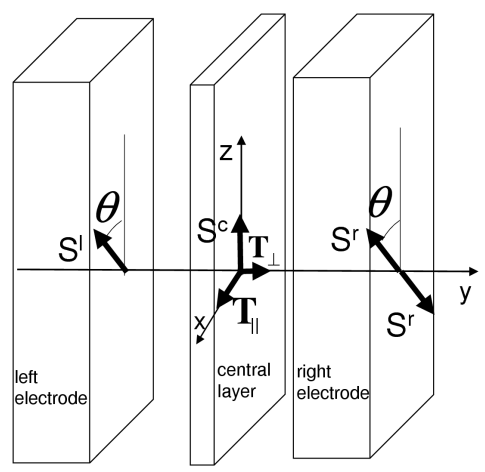

Fig. 1. Schematic of the system considered in this paper. $S^{\mathrm{l}}, S^{\mathrm{c}}$ and $S^{\mathrm{r}}$ denote the net spin in the left, central and right layers, respectively, whereas $T_{\|}$and $T_{\perp}$ denote the in-plane and normal spin torque components.

Generally, the spin torque exerted on the central layer has two components, the in-plane and out-of-plane (also called normal) ones. The in-plane torque $T_{\|}$lies in the 
plane common to the net spin of all magnetic layers, whereas the normal one $T_{\perp}$ is perpendicular to this plane. Both components of the spin torque are calculated as a function of the angle $\theta$ formed by magnetic moments in the left and central layers and as a function of the central layer thickness $d_{\mathrm{c}}$. Calculations have been performed in the zero temperature limit and in the free-electron-like one-band model [7]. Accordingly, the electronic structure of ferromagnetic layers is modelled by a parabolic spinsplit band with the free electron mass. Non-collinear orientation of magnetic moments in different layers requires spinor transformations in the description of the matching boundary conditions for the electron wave function $[1,7]$.

In the coherent tunneling model assumed here the tunneling electron is in a coherent state of the whole system consisting of three magnetic layers and two barriers. The spin torque components have been then determined from the conservation of the total angular momentum, i.e. momentum of localized moments and that carried by spin current associated with free electrons. Accordingly, the in-plane torque is calculated from the formula

$$
T_{\|}=\frac{\hbar}{2}\left(J_{x}^{\mathrm{sl}}-J_{x}^{\mathrm{sr}}\right),
$$

whereas the normal torque is given by

$$
T_{\perp}=\frac{\hbar}{2}\left(J_{y}^{\mathrm{sl}}-J_{y}^{\mathrm{sr}}\right),
$$

where $J_{\mu}^{\text {sl }}$ denotes the $\mu$-th component $(\mu=x, y)$ of the spin current density calculated in the central layer at a point located near the interface with the left barrier, whereas $J_{\mu}^{\text {sr }}$ denotes analogous density calculated at the interface with the right barrier. The $\mu$-th component of the spin current corresponding to electrons described by the wave function $\Psi(y)$ is calculated from the general expression

$$
\begin{gathered}
j_{\mu}^{\mathrm{s}}(y)=\frac{\mathrm{i} \hbar}{2 m}\left[\left(\frac{\partial}{\partial y} \Psi^{+}(y)\right) \sigma_{\mu} \Psi(y)\right. \\
\left.-\Psi^{+}(y) \sigma_{\mu} \frac{\partial}{\partial y} \Psi(y)\right],
\end{gathered}
$$

where $\sigma_{\mu}$ are the Pauli matrices, whereas $\Psi(y)$ has the spinor form in the relevant local reference frame. To find the total spin currents $J_{\mu}^{\mathrm{sl}}$ and $J_{\mu}^{\mathrm{sr}}$, one has to sum contributions from electrons of different in-plane wave vectors and energies. In the calculations of normal torque, the contribution from electrons in the energy window between the Fermi levels of both external electrodes, as well as the contribution from electrons of lower energy (below tunnel window) are taken into account. It is assumed that the charge current due to a positive bias applied to the junction corresponds to electrons tunneling from the left to right electrodes. Electrons of lower energy do not contribute to the charge current and in-plane torque, but they contribute to the out-of-plane torque and this contribution can be significant especially in the systems with small bias voltage applied to the junction. In particular, this contribution is responsible for the exchange coupling between ferromagnetic layers, which appears also for vanishing bias voltage.

\section{Results and discussion}

Numerical calculations have been performed for the parameters corresponding to systems with semiconductor ferromagnetic electrodes: the Fermi energy $E_{\mathrm{F}}=0.1 \mathrm{eV}$, the spin splitting of the electron band $2 \Delta=0.12 \mathrm{eV}$, the barrier height $U=0.1 \mathrm{eV}$ and the barrier thickness $d=2.0 \mathrm{~nm}$. In Fig. 2 the torque components are

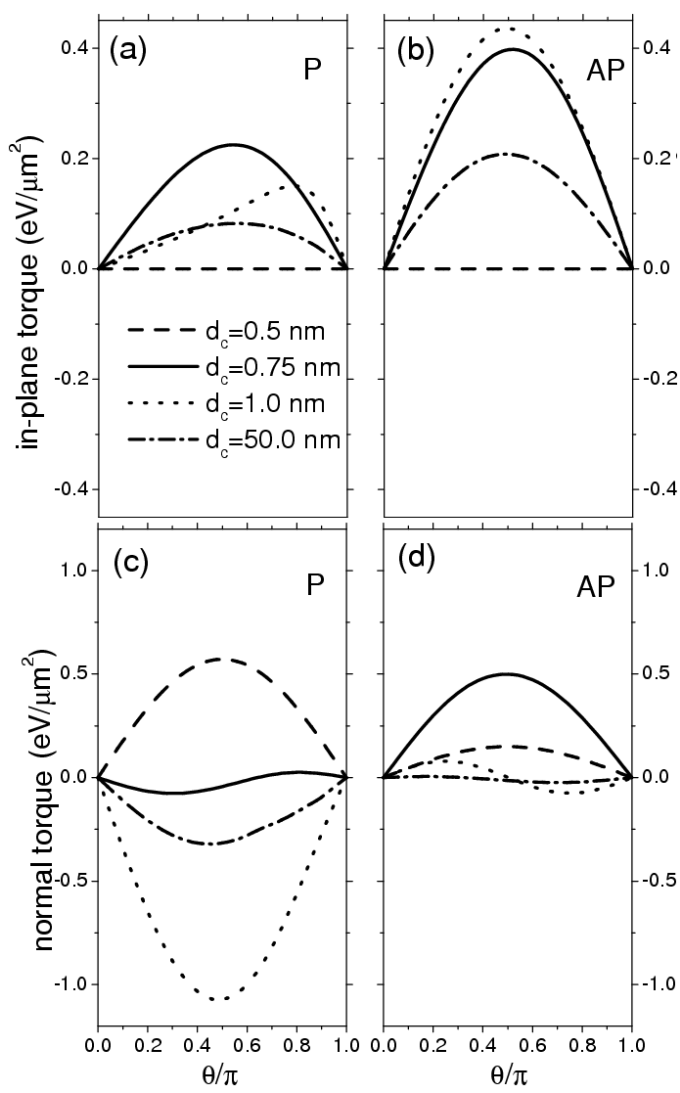

Fig. 2. The in-plane ( $a$ and b) and normal (c and d) components of the spin torque calculated as a function of the angle $\theta$ for the parallel (a and c) and antiparallel (b and $d$ ) orientation of the magnetic moments in the external electrodes. The results are for the four indicated values of the central layer thickness $d_{\mathrm{c}}$. The other parameters are: the Fermi energy $E_{\mathrm{F}}=0.1 \mathrm{eV}$, spin splitting of the electron band $2 \Delta=0.12 \mathrm{eV}$, the bias voltage $V=0.02 \mathrm{~V}$, the barrier height $U=0.1 \mathrm{eV}$, and barrier thickness $d=2.0 \mathrm{~nm}$.

presented as a function of the angle $\theta$ for the bias voltage $V=0.02 \mathrm{~V}$ and for four different values of $d_{\mathrm{c}}$. As one can see, the obtained results strongly depend on the central layer thickness $d_{c}$. The in-plane torque has the same sign for all values of $d_{\mathrm{c}}$, but its magnitude depends on $d_{c}$. The normal torque obtained for different $d_{c}$ can also differ in sign. Both torque components vanish in the collinear configurations. The in-plane torque is positive for $0<\theta<\pi$ and usually achieves a maximum value for $\theta$ close to $\pi / 2$, similarly as in single junctions [7]. However, position of the maximum depends on the cen- 
tral layer thickness and for certain values of $d_{\mathrm{c}}$ can differ from $\theta=\pi / 2$. The $\theta$ dependence of the normal torque is more complicated. In the antiparallel (AP) configuration in junctions with $d_{\mathrm{c}}=1.0 \mathrm{~nm}$, the torque changes sign for $\theta$ close to $\pi / 2$, so it vanishes for $\theta$ corresponding to the non-collinear configuration. Similar behavior is obtained in the parallel $(\mathrm{P})$ geometry for $d_{\mathrm{c}}=0.75 \mathrm{~nm}$. However, the magnitude of the normal torque in junctions with such anomalous angular dependence is generally smaller than in other junctions.
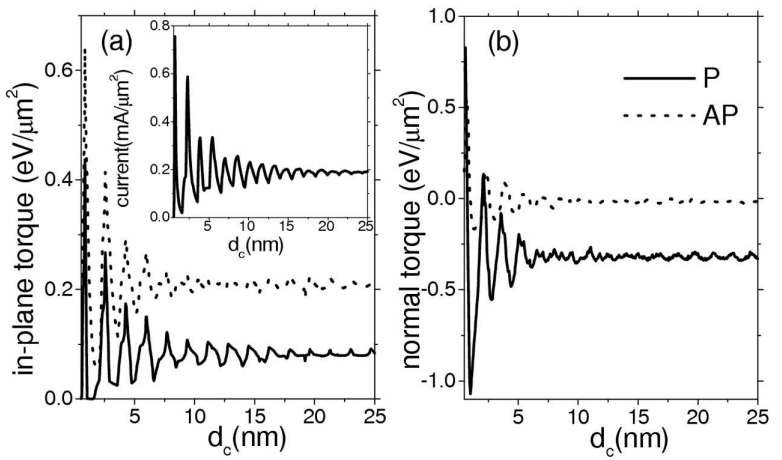

Fig. 3. The in-plane (a) and normal (b) components of the spin torque in the $\mathrm{P}$ and AP configuration, calculated as a function of the central layer thickness for $\theta=\pi / 2$. The inset shows the analogous dependence obtained for the charge current. The other parameters of the junction are the same as in Fig. 2.

To demonstrate the strong influence of the central layer thickness on the spin torque we plot in Fig. 3 both torque components versus $d_{\mathrm{c}}$, calculated for $\theta=\pi / 2$ and for two different magnetic configurations. As one can see again, both components of the torque in both configurations strongly depend on $d_{c}$. In junctions with special thickness of the central layer, a considerable enhancement of the spin torque can be observed. Similar oscillatory-type behavior can be observed in the plot of the charge current versus $d_{\mathrm{c}}$ (inset in Fig. 3a). Such a dependence results from the presence of resonant states formed in the central layer, which have a significant influence on the transport properties of double tunnel junctions. Despite the complex character of the presented dependence, one can notice that the oscillations are damped in junctions with a relatively thick central layer. In such junctions both torque components tend to constant values. Comparing the torques in $\mathrm{P}$ and $\mathrm{AP}$ configurations, one can note that the magnitude of the in-plane torque is larger in the antiparallel configuration than in the parallel one.
The opposite relation is observed for the normal torque component. In the AP configuration the normal torque is close to zero in junctions with a relatively thick central layer. This can be explained by the analysis of the torque acting on magnetic moment of the central layer as a sum of two torques exerted by electrons tunneling through the left and right barriers. In the case of the normal torque these two contributions have the same signs in the $\mathrm{P}$ configuration and opposite signs in the AP configuration. These signs are in agreement with the signs of the torques exerting on the sink and the source electrode in the single junction for the appropriate geometry of magnetic moments. As both contributions are of comparable magnitude, the strong difference between normal torques in the $\mathrm{P}$ and the AP configuration is observed and the normal torque in the AP configuration is very small. On the other hand, for the in-plane torque (in the limit of small bias voltage) both contributions have the opposite signs in the $\mathrm{P}$ configuration and the same signs in the AP configuration. This explains why the in-plane torque is larger in the $\mathrm{AP}$ than in the $\mathrm{P}$ configuration. However, the magnitude of the in-plane torque exerted by electrons tunneling through the left barrier is higher than the one exerted by electrons tunneling through the right barrier. As a result, the in-plane torque in $\mathrm{P}$ configuration is higher than the normal torque in AP configuration in junctions with a thick central layer.

\section{Acknowledgments}

This work was supported by funds of the Ministry of Science and Higher Education as a research project in years 2006-2009.

\section{References}

[1] J.C. Slonczewski, Phys. Rev. B 71, 024411 (2005); Phys. Rev. B 39, 6995 (1989).

[2] Y. Huai, F. Albert, P. Nguyen, M. Pakala, T. Valet, Appl. Phys. Lett. 84, 3118 (2004).

[3] D. Chiba, Y. Sato, T. Kita, F. Matsukura, H. Ohno, Phys. Rev. Lett. 93, 216602 (2004).

[4] A. Vedyayev, N. Ryzhanova, B. Dieny, N. Strelkov, Phys. Lett. A 355, 243 (2006).

[5] I. Theodonis, A. Kalitsov, N. Kioussis, Phys. Rev. B 76, 224406 (2007).

[6] I. Theodonis, A. Kalitsov, N. Kioussis, J. Magn. Magn. Mater. 310, 2043 (2007).

[7] M. Wilczyński, J. Barnaś, R. Świrkowicz, Phys. Rev. B 77, 054434 (2008). 